\title{
Transitional behavior of asymmetric oscillatory boundary layers
}

\author{
By Ahmad SANA ${ }^{1}$, Hitoshi TANAKA ${ }^{2}$ and Hiroto YAMAJI ${ }^{3}$
}

\begin{abstract}
A simple and inexpensive piston mechanism has been used to generate asymmetric oscillatory motion in an oscillating tunnel with smooth walls and detailed velocity measurement has been performed by one component LDV under transition from laminar to turbulent flow. The experimental data for mean and fluctuating velocity is presented and comparison has been made with $k-\epsilon$ model prediction. The turbulence generation during deceleration and its distribution in cross-stream direction during acceleration has found to be qualitatively similar to sinusoidal case. Finally, a friction factor diagram has been proposed for asymmetric oscillatory boundary layers by using the model prediction.
\end{abstract}

Keywords: boundary layer, asymmetric oscillation, $k-\epsilon$ model.

\section{Introduction}

In natural coastal environments, the wave profiles are generally asymmetric due to the non-linear effects. A precise estimation of sediment transport under actual field conditions, therefore requires an adequate knowledge of the properties of asymmetric oscillatory boundary layers. A number of experimental studies have been performed in open flume by various researchers, e.g. Kuo and Chen(1990). But it is difficult to produce a turbulent asymmetric oscillatory boundary layer in an open flume having an adequately thick boundary layer in order to perform detailed measurement. On the other hand, it is possible to generate asymmetric oscillation in an oscillating tunnel, to produce sufficiently high Reynolds numbers and a well defined boundary layer. The asymmetry produced in this case is though, due to the imposed pressure gradient, because the continuity condition eliminates the non-linear (convection) term from the equation of motion. But to impose the pressure gradient in order to generate an asymmetric oscillation with the help of piston mechanism is also not an easy task. It requires a highly expensive sophisticated equipment to control the piston movement, the reason being for the scarcity of the experiments in this regard. Nadaoka et al. $(1994,1996)$ and Ribberink and Al-Salem(1995) have performed some experiments by using computer controlled piston systems to study the characteristics of asymmetric oscillatory boundary layers.

Recently, Tanaka et al.(1996) proposed a rather simple and inexpensive piston mechanism, by which the asymmetric oscillation is produced mechanically. They performed the experiment under laminar flow to validate this system.

In the present study, this system has been employed to study the properties of asymmetric oscillatory boundary layers on a smooth bottom at higher Reynolds numbers. The numerical prediction for the present cases, which belong to transitional regime between laminar and turbulent flow, has been done by the low Reynolds number $k-\epsilon$ model proposed by Jones and Launder(1972). In case of sinusoidal oscillatory boundary layers, this model has found to be quite efficient (see Justesen, 1988). Especially, for the prediction of transitional properties in the sinusoidal boundary layers, this model has found to be superior to a number of contemporary models of this type, as shown by Tanaka and Sana(1994) and Sana and Tanaka(1996).

\section{Experimental Conditions}

The schematic description of the piston mechanism to generate asymmetric oscillatory motion proposed by Tanaka et al.(1996) has been shown along with the oscillating tunnel in Fig.1. A detailed measurement of velocity was carried out by using one component LDV, and the data analysis was done offline on a PC.

\footnotetext{
${ }^{1}$ M. Eng. Research Associate, Department of Civil Eng. Tohoku University, Aoba-ku, Sendai 980-77

${ }^{2}$ D. Eng. Professor, ditto

${ }^{3}$ Lab. Assistant, ditto
} 


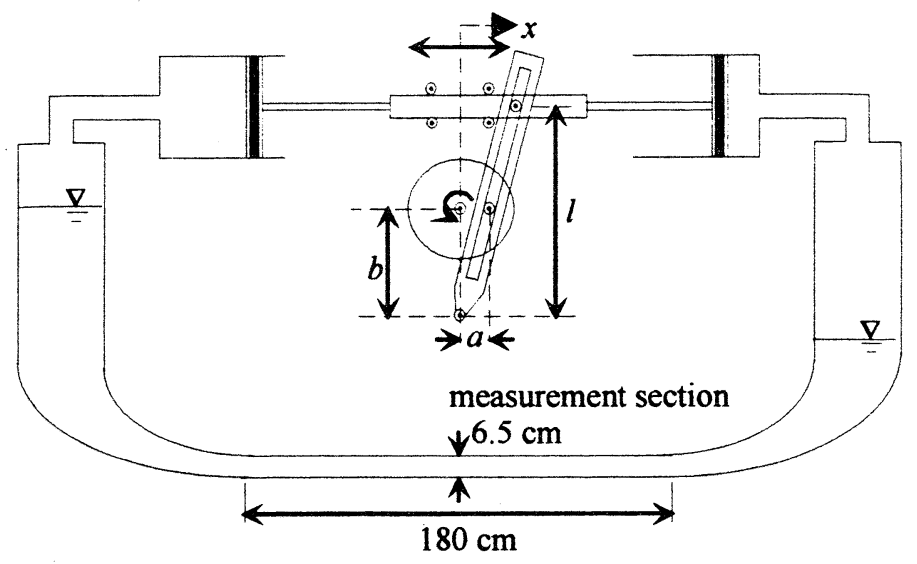

Fig.1 Schematic description of piston movement system and the oscillating tunnel.

Tanaka et al.(1996) have shown theoretically that the oscillation generated by the present piston movement system produces an oscillation similar to the one obtained from cnoidal wave theory and by changing the dimensions $a, b$ and $l$ of the system, asymmetry of the wave profile may be changed. It was found that with air as working fluid, the temporal velocity variation at the axis of symmetry of the tunnel showed excellent agreement with the cnoidal wave theory, however, due to the restricted length of the tunnel, the use of water as working fluid was inevitable to achieve sufficiently high Reynolds numbers. But in that case, it was necessary to allow the free passage of air into and out of the vertical risers of the tunnel to get the velocity at axis of symmetry in close agreement with the theory, because the performance of the piston movement system under high pressure was not good. Even then perfect agreement with the theory could not be achieved as may be observed from Fig.2, showing the velocity at axis symmetry for Case N04 and N02.

Table 1 shows the experimental conditions for the cases presented herein. In this table, degree of asymmetry $A_{s}=U_{c} /\left(U_{c}+U_{t}\right)$, crest Reynolds number $R e_{c}=U_{c} \delta_{l c} / \nu$, trough Reynolds number $R e_{t}=U_{t} \delta_{l t} / \nu$, and $U_{c}$ and $U_{t}$ are velocity magnitudes at crest and trough, respectively, $\nu$ being the kinematic viscosity. The Stokes' layer thicknesses and time periods for crest and trough are given as $\delta_{l c}\left(=\sqrt{2 \nu t_{c} / \pi}\right), \delta_{l t}\left(=\sqrt{2 \nu t_{t} / \pi}\right), t_{c}$ and $t_{t}$, respectively. The definitions of $R e_{c}$ and $R e_{t}$ have been adopted from Nadaoka et al.(1994). For the numerical computations the values of $R E_{c}=U_{c}^{2} / \omega \nu$ and $S=U_{c} /\left(\omega y_{h}\right)(\omega=2 \pi / T, T=$ period of oscillation $)$ are also provided.

Table 1. Experimental conditions for asymmetric oscillatory boundary layer experiments

\begin{tabular}{|l|c|c|c|c|c|c|c|c|c|c|}
\hline Exp. & $T(\mathrm{sec})$ & $t_{c}(\mathrm{sec})$ & $t_{t}(\mathrm{sec})$ & $U_{c}(\mathrm{~cm} / \mathrm{s})$ & $U_{t}(\mathrm{~cm} / \mathrm{s})$ & $A_{s}$ & $R e_{c}$ & $R e_{t}$ & $R E_{c}$ & $S$ \\
\hline N02 & 2.00 & 0.84 & 1.16 & 109.4 & 66.9 & 0.62 & 859 & 616 & $4.38 \times 10^{5}$ & 10.72 \\
\hline N03 & 2.38 & 0.98 & 1.40 & 89.3 & 56.0 & 0.61 & 744 & 559 & $3.40 \times 10^{5}$ & 10.41 \\
\hline N04 & 3.92 & 1.66 & 2.26 & 55.6 & 42.7 & 0.57 & 604 & 541 & $2.17 \times 10^{5}$ & 10.67 \\
\hline
\end{tabular}
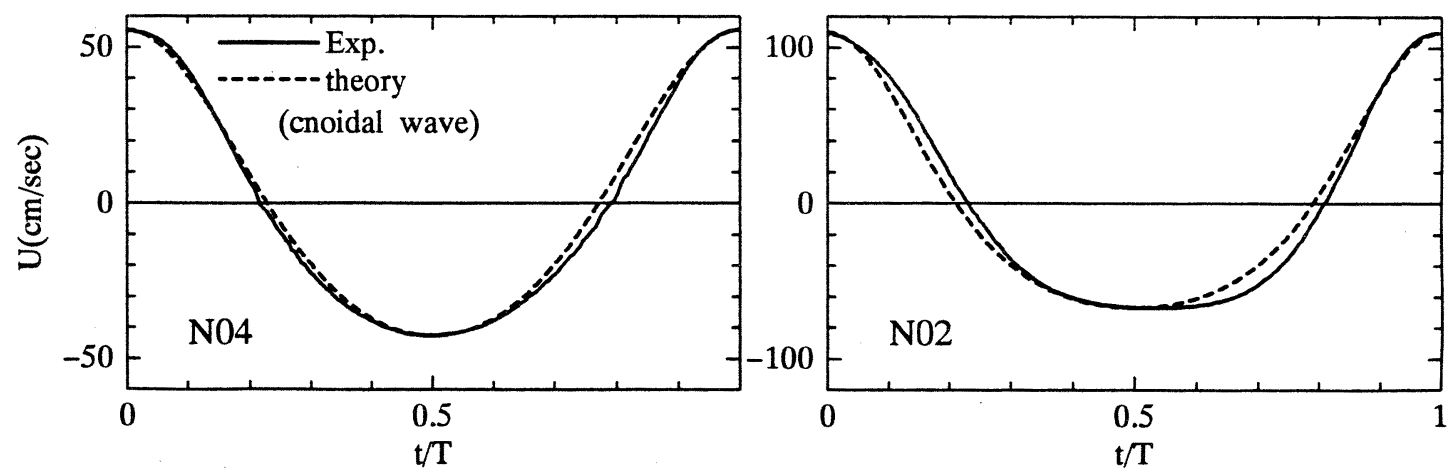

Fig.2 Velocity at axis of symmetry for Case N04 and N02. 


\section{$3 \quad k-\epsilon$ Model}

In the present study, the numerical prediction was done by using the original version of low Reynolds number $k-\epsilon$ model by Jones and Launder (1972), the governing equations in dimensionless form are expressed by Sana and Tanaka(1996). For a particular case, these equations require only Reynolds number $R E_{c}$ and $S$ values to provide the solution in dimensionless form.

A Crank-Nicolson type implicit finite difference scheme was employed. In order to achieve better accuracy near the wall, the grid spacing was allowed to increase exponentially. In space 100 and in time 6000 steps per wave cycle were used. The nonlinear governing equations were solved by iteration method. The convergence limit was set to $5 \times 10^{-5}$. The detail in this regard may be found elsewhere (e.g. see Sana and Tanaka, 1996).

\section{Results and Discussion}

\subsection{Velocity profile}

For Case N04 it may be noted that the Reynolds number in this case $\left(R e_{c}=604, R e_{t}=541\right)$ is at the beginning of transitional range because the value of critical Reynolds number for sinusoidal oscillatory boundary layers is 550, as given by Hino et al.(1976). Figure 3 shows the cross-stream velocity profile at selected phases. The velocity overshooting at all the phases is stretched in cross-stream direction in comparison with the laminar velocity profiles, which shows the generation of turbulence, though small, causing an increased momentum transport from high velocity regions in cross-stream dimension. The laminar velocity profiles plotted here are as per theory described by Tanaka et al.(1996). The velocity profile for Case N04 shows a good agreement with the $k-\epsilon$ model prediction just at the beginning of deceleration phase $(\mathrm{t} / \mathrm{T}=0.0)$, especially where the velocity overshooting occurs. But during the course of deceleration, it seems that the model fails to cope with the flow situations. A similar discrepancy was found in case of sinusoidal oscillatory boundary layers also, but in that case, during the deceleration phase, pressure gradient is not so steep as in the present asymmetric case. That is why, in sinusoidal case, the disagreement with the experimental data is not pronounced (see e.g. Tanaka and Sana, 1994 and Sana and Tanaka, 1996).

By using Jensen's definition, according to which the boundary layer thickness $\delta$ is the distance from the wall to the location of maximum cross-stream velocity at $\omega t=0$ (or at $\omega t=T / 2$ ), it may be shown that the boundary layer thickness $\delta$ is proportional to the Stokes' layer thickness $(=\sqrt{\nu T / \pi})$, which suggests that the value of $\delta$ must be greater under trough than that under the crest due to longer period of time contained in the trough. The result may be observed at $t / T=0.5$ where the boundary layer thickness is greater than that at $t / T=0$ from the velocity profiles presented herein. The degree of asymmetry in Case N04 is not so high as may be observed from the value of $A_{s}(=0.57)$ in this case. That is why the difference between boundary layer thickness under crest and trough is not so significant.

Under higher Reynolds number in case N02, the deviation of the experimental data from laminar velocity profile is more obvious (Fig.4). The diminished velocity overshooting and the stretching in cross-stream direction due to high momentum transport resulting from higher turbulence production is evident here also. The velocity near the wall increases so that a logarithmic layer is well defined at all the phases except near the flow reversal. The inability of the model to predict the velocity profile in deceleration phase is evident in this case also. Moreover, it may be observed that the discrepancy between the model prediction and experimental data near the wall during acceleration also has increased. Since the degree of asymmetry is higher $\left(A_{s}=0.62\right)$ as compared to Case N04, therefore, the difference of boundary layer thickness under crest and trough is also greater.

\subsection{Turbulence intensity}

In a manner similar to that in sinusoidal oscillatory boundary layers, the turbulence is produced in near wall region during deceleration phase as may be observed from Fig.5 at $t / T=0.0-0.2$ and $t / T=0.5-0.7$ and it is distributed in cross-stream direction during acceleration phase $(t / T=0.3-0.4,0.8-0.9)$. In this case the Reynolds number is close to the critical value as mentioned before, that is why, the turbulence intensity is not so high. Consequently, the cross-stream peak values of $u^{\prime}$ are located rather far from the wall.

The $k-\epsilon$ model provides the turbulent kinetic energy from which the fluctuating velocity in $x$ direction $u^{\prime}$ has been computed from the approximate formula; $u^{\prime}=1.052 \sqrt{k}$ (Nezu, 1977) in order to compare with the experimental data. The comparison presented here for $u^{\prime}$ therefore depends on the degree of accuracy of this approximation as well. For Case N04, the model prediction far from the wall is generally good (Fig.5). In the near wall region, the shape of $u^{\prime}$ profile and the peak value shows some discrepancy. As the Reynolds number gets higher in Case N02 the turbulence intensity increases (Fig.6). 

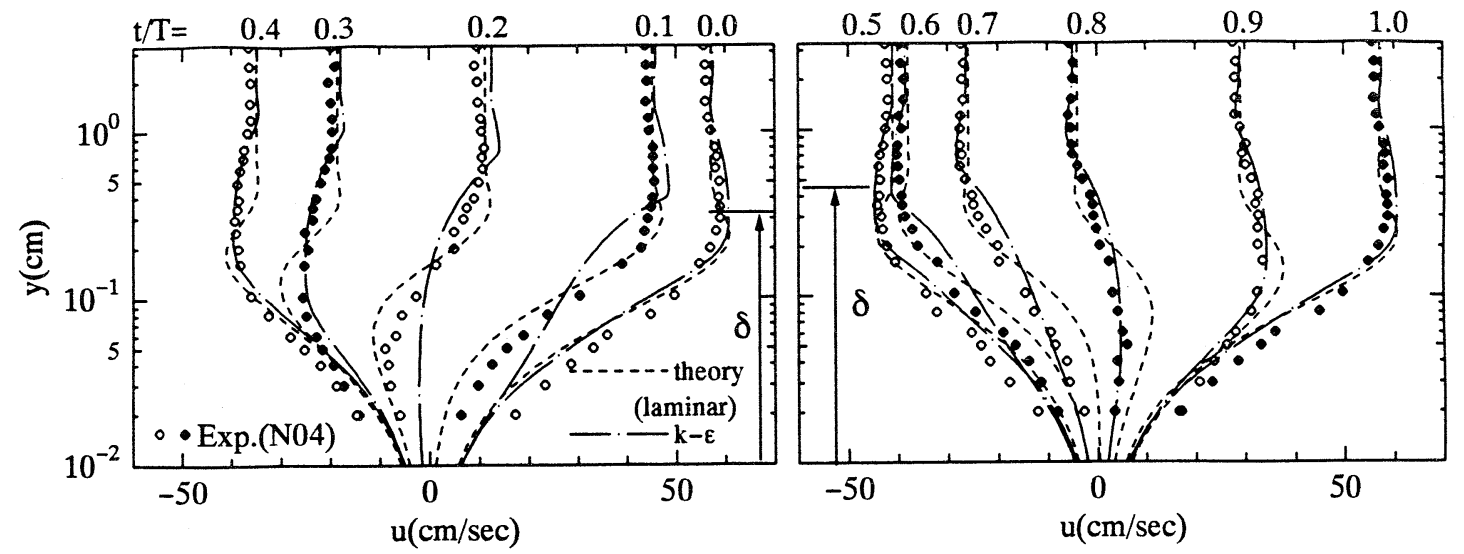

Fig.3 Velocity profile for Case N04.

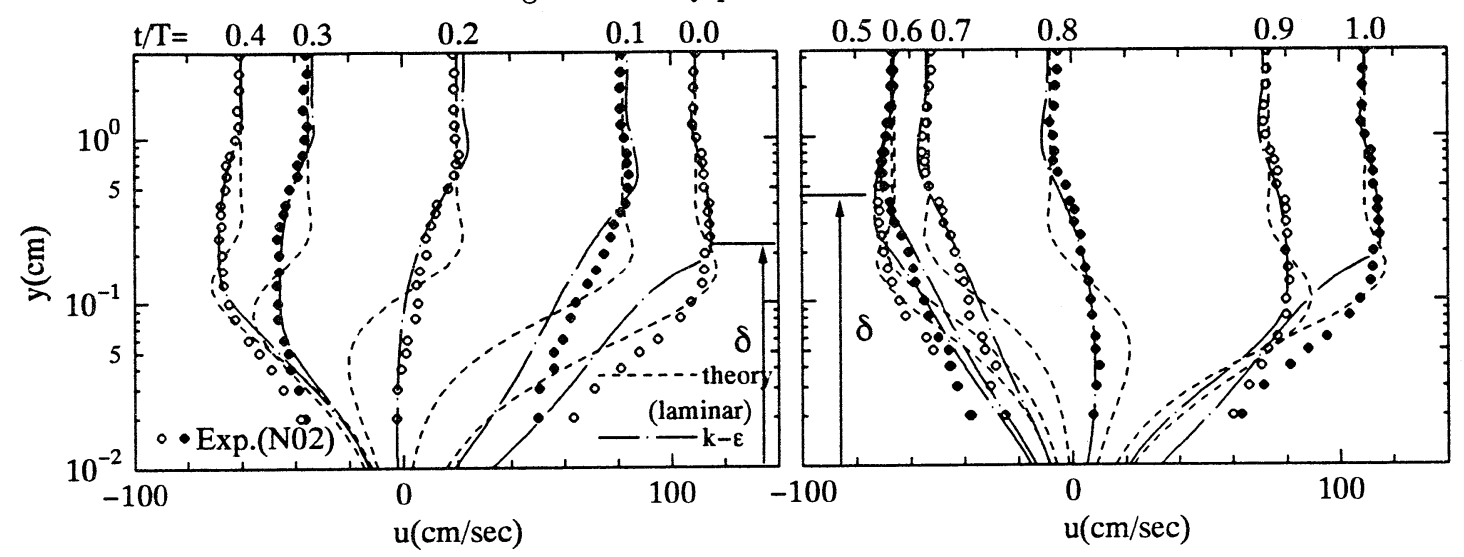

Fig.4 Velocity profile for Case N02.

The peak values of $u^{\prime}$ are located nearer the wall under crest and trough as compared to those at corresponding phases in Case N04. The performance of the model in predicting $u^{\prime}$, in this case, is better than in the previous case. The prediction of peak value of $u^{\prime}$ close to the wall is not satisfactory in this case as well, at some phases.

\section{Friction Factor}

In order to compute bottom shear stress from the free-stream velocity, knowledge of the friction factor is important from practical point of view. For sinusoidal oscillatory boundary layers a comprehensive data set is available over a wide range of Reynolds numbers, so that the friction factor diagram may be drawn. On the contrary, for asymmetric uscillatory boundary layers, not only experimental data is scarce, but the studies based on analytical and numerical models are also very few. In the present study, a friction factor diagram has been generated by using the low Reynolds number $k-\epsilon$ model in order to visualize the tentative variation of friction factor with Reynolds number and degree of asymmetry.

It is difficult to define a single friction factor for asymmetric boundary layers, that is why following Tanaka, Sumer and Fredsøe(1996), the crest and trough friction factors have been defined separately as; $f_{w c}=$ $2.0 \tau_{0 c} /\left(\rho U_{c}^{2}\right)$ and $f_{w t}=2.0 \tau_{0 t} /\left(\rho U_{c}^{2}\right)$, where, $\tau_{0 c}$ and $\tau_{0 t}$ are maximum values of wall shear stress in crest and trough periods respectively.

This diagram (Fig.7) shows that the difference between crest values of friction factor in laminar as well as in turbulent flow, with the change in degree of asymmetry, is not so significant. But in trough phase $A_{s}$ value creates a considerable difference at a certain Reynolds number. Another interesting feature of this figure is that the transition from laminar to turbulent flow is delayed by virtue of the Reynolds number with the increase in $A_{s}$ value. The expression for friction factor in case of laminar sinusoidal boundary layer $\left(f_{w}=2 /(R E)^{1 / 2}\right)$ has also been plotted and shows an excellent agreement with asymmetric case at $A_{s}=0.5$. 


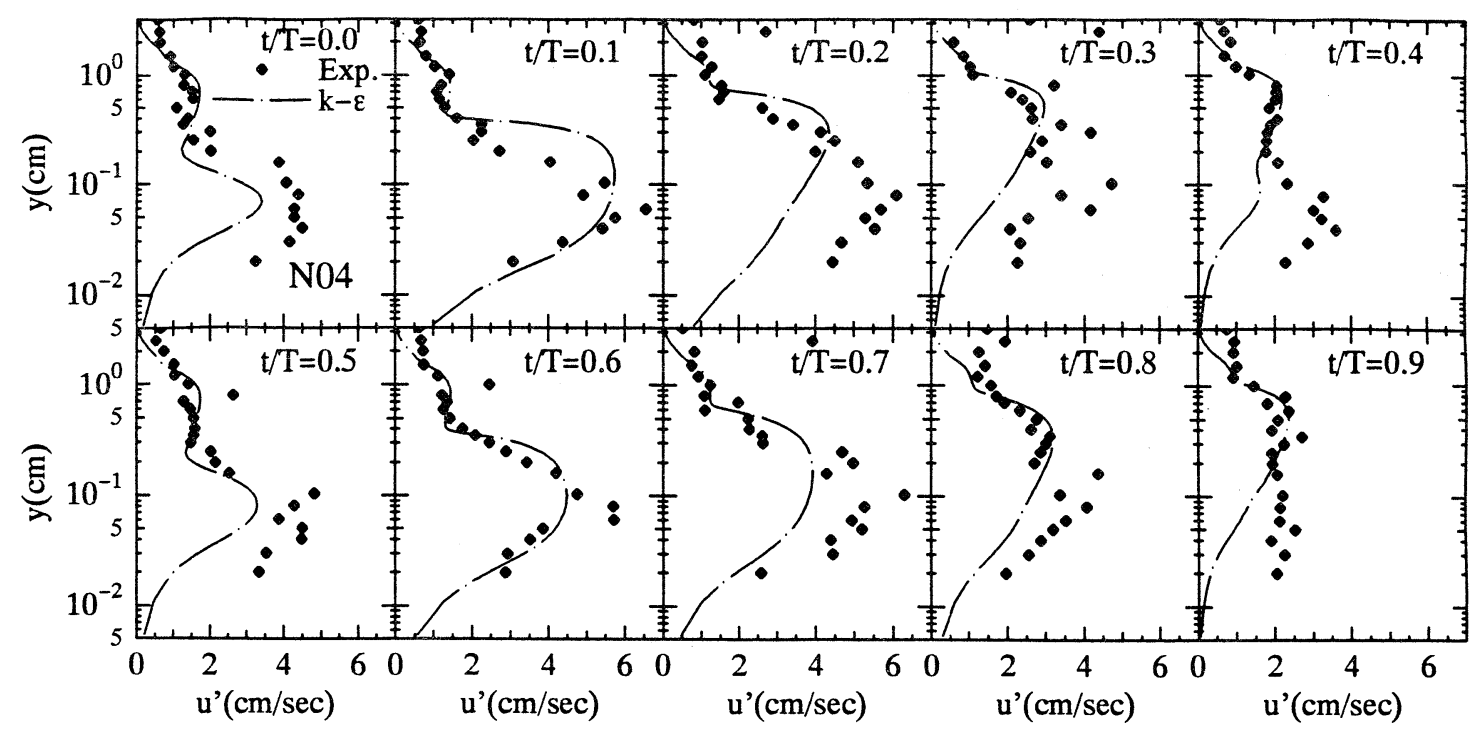

Fig.5 $x$-direction fluctuating velocity for Case N04.

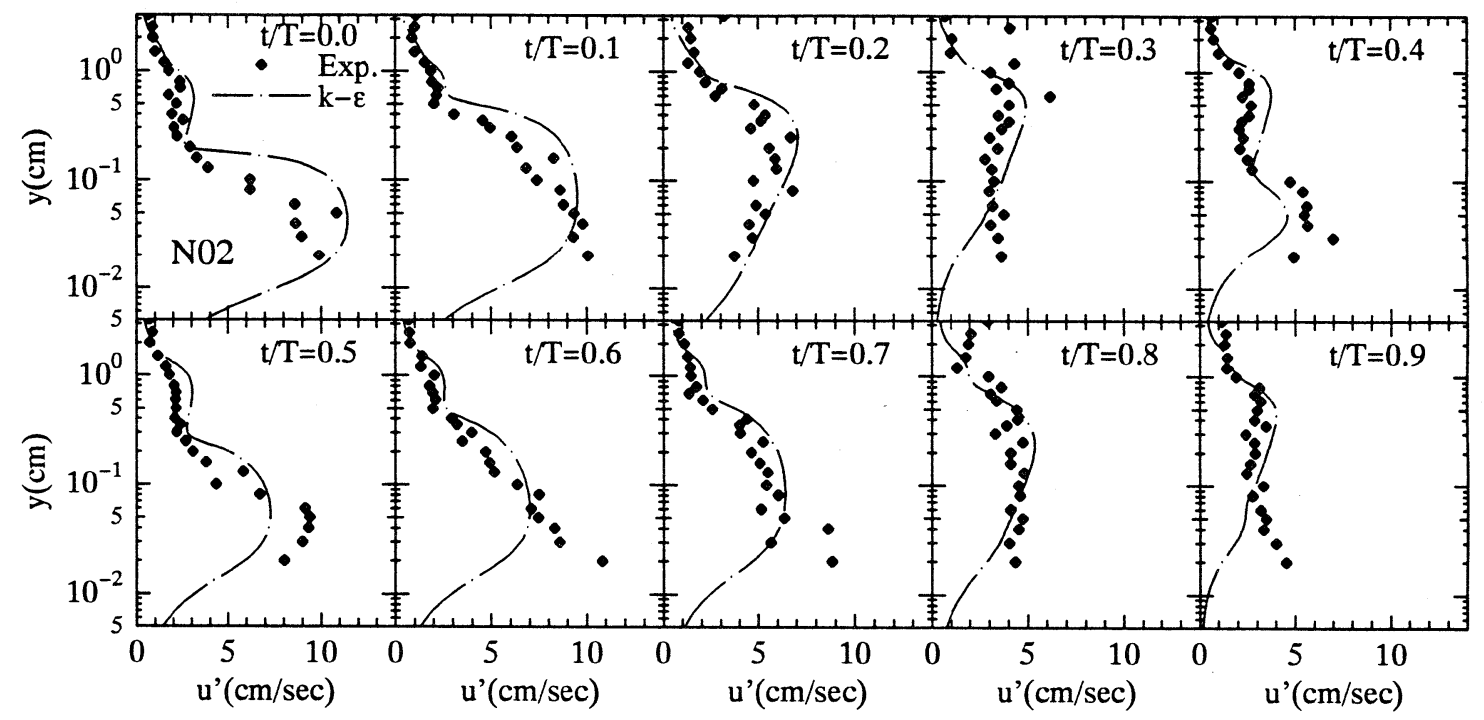

Fig.6 $\quad x$-direction fluctuating velocity for Case N02.

The friction factor for the present experimental data was computed from the wall shear stress profiles obtained by integrating the equation of motion from the wall to axis of symmetry. As may be observed from this figure that the three experiments lie within the transitional region.

The qualitative agreement of the model prediction with the experimental data is satisfactory for all the three cases. Although for Case N04 which corresponds to the beginning of transition, the model underestimates the friction factor at crest and trough, however, the second data set from the left hand side(N03) shows quite good agreement with the model prediction. For the data set having highest Reynolds number(N02) the model overestimates both the values of $f_{w c}$ and $f_{w t}$. As may be observed from Fig. 7 that three of the experiments with $A_{s} \simeq 0.6$ depict a gradual transition process similar to that observed in case of sinusoidal oscillatory boundary layers (see e.g. Jensen, 1989). On the other hand, the model shows rather abrupt transition from laminar to turbulent flow.

In most of the field situations the flow is turbulent, consequently the part of friction factor diagram relevant to fully turbulent flow is very important. But the experimental data for higher Reynolds numbers are not available as yet, that is why it is difficult to draw the final conclusion regarding the capabilities of the model in fully turbulent regime for asymmetric oscillatory boundary layers. 


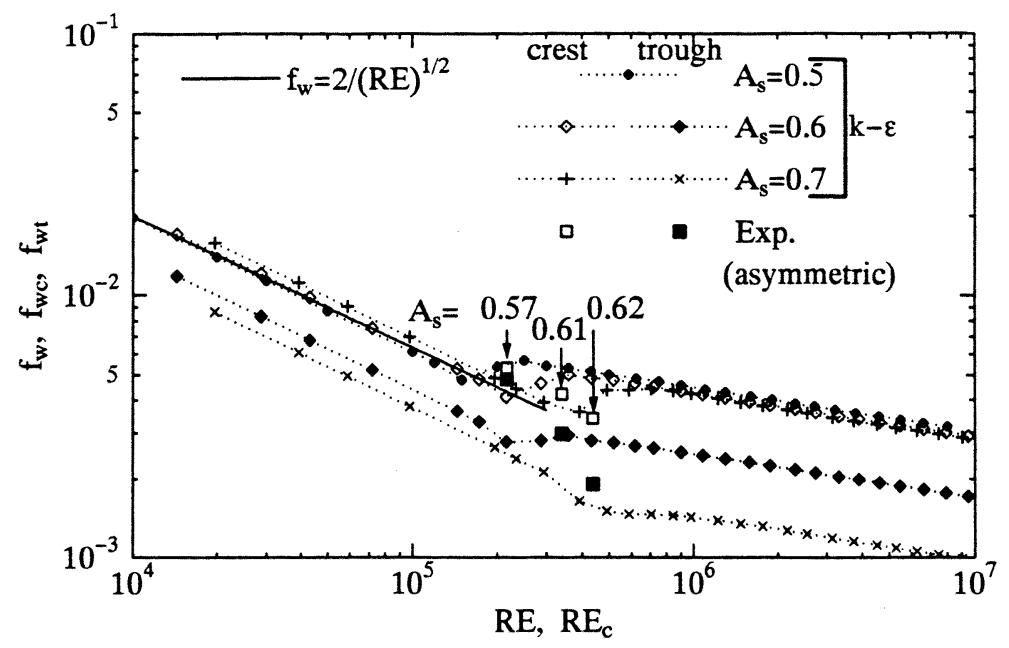

Fig.7 Friction factor diagram for asymmetric oscillatory boundary layers.

\section{Conclusion}

An inexpensive piston movement system has been employed to generate asymmetric oscillatory motion in an oscillating tunnel. The critical Reynolds number for sinusoidal oscillatory boundary layer transition is valid for the asymmetric oscillatory boundary layers with small degree of asymmetry values. The low Reynolds number $k-\epsilon$ model by Jones and Launder(1972) showed good performance to predict mean velocity profile during acceleration phase, but during deceleration phase its predictions were not satisfactory. The overall agreement between the model prediction and experimental data is satisfactory for $x$ direction fluctuating velocity. The friction factor diagram produced by the low Reynolds number $k-\epsilon$ model shows that with the change in degree of asymmetry, crest friction factor does not show considerable variation, whereas trough values vary a lot. In order to draw the final conclusion in this regard, the experiments under fully turbulent asymmetric oscillatory boundary layers are required.

\section{Acknowledgment}

The authors gratefully acknowledge the financial support as Grant-in-aid by The Ministry of Education, Science and Culture, Japan for this study.

\section{References}

Hino, M., Sawamoto, M. \& Takasu, S. 1976 J. Fluid Mech. 75, 193-207.

Jensen, P. 1989 Experimental Investigation of Turbulent Oscillatory Boundary Layers, Series Paper No.45, ISVA, Tech. Univ. Denmark.

Jones, W. P. \& Launder, B. E. 1972 Int. J. Heat Mass Transfer 15, 301-314.

Justesen, P. 1988 Turbulent Wave Boundary Layers, Series Paper No.43, ISVA, Tech. Univ. Denmark.

Kuo, C. T. \& Chen, W. J. 1990 In Proc. 22nd Int. Conf. Coastal Eng. 1637-646.

Nadaoka, N., Yagi, H., Nihei, Y. \& Nomoto, K. 1994 In Proc. Japanese Conf. Coastal Eng. 41, 141-145.

Nadaoka, N., Nihei, Y., Yagi, H. \& Nomoto, K. 1996 In Proc. Japanese Conf. Coastal Eng. 43, 441-445.

Nezu, I. 1977 Turbulent structure in open channel flows. Ph. D. Dissertation, Kyoto Univ., Japan.

Ribberink, J. S. \& Al-Salem, A. 1995 Coastal Eng. 25, 205-225.

Rodi, W. \& Scheuerer, G. 1986 J. Fluids Eng., Trans. ASME 108, 174-179.

Sana, A. \& Tanaka, H. 1996 In Flow Modeling and turbulence measurements 6, 363-370.

Tanaka, H. \& Sana, A. 1994 In Sediment Transport Mechanism in Coastal Environments and Rivers, 14-25.

Tanaka, H., Sumer, B. M. \& Fredsøe, J. 1996 J. Hyd. Coastal Environment Eng. (JSCE), (submitted).

Tanaka, H., Yamaji, H., Sana, A. \& Shuto, N. 1996 J. Hyd. Coastal Environment Eng. (JSCE), (accepted). 